\title{
Enrichment Paradox Induced by Spatial Heterogeneity in a Phytoplankton - Zooplankton System
}

\author{
J.-C. Poggiale ${ }^{a, 1}$, M. Gauduchon ${ }^{a}$ and P. Auger $^{b}$ \\ ${ }^{a}$ Centre d'Océanologie de Marseille, UMR CNRS 6117 \\ Laboratoire de Microbiologie, de Géochimie et d'Ecologie Marines \\ Université de la Méditerranée, Campus de Luminy Case 901 \\ 13288 Marseille Cedex 9 - France \\ ${ }^{b}$ UR GEODES - IRD Bondy - 32, Av Henri Varagnat 93143 Bondy Cedex - France
}

\begin{abstract}
This paper is devoted to the study of a predator-prey model in a patchy environment. The model represents the interactions between phytoplankton and zooplankton in the water column. Two patches are considered with respect to light availability: one patch is associated to the surface layer, the second patch describes the bottom layer. We show that this spatial heterogeneity may destabilize the predator-prey system, even in oligotrophic system where the nutrient is low enough to avoid "paradox-enrichment" phenomenon. Indeed, in this case, an heterogeneity index can be used as a bifurcation parameter, leading to a Hopf bifurcation. Moreover, we assume that individuals can be dispersed in both patches via hydrodynamism processes, like in a mixed layer. The effect of mixing intensity is analysed as well as interactions between dispersion and enrichment. We also show that, in some cases, spatial heterogeneity has a stabilizing effect. These contrasted results are examined by considering the non linear interaction between heterogeneity, dispersal and enrichment and some mechanisms leading to stabilization/destabilization are exhibited.
\end{abstract}

Key words: spatial heterogeneity, enrichment paradox, singular perturbations, bifurcations AMS subject classification: 92D25, 58F35, 34A47, 92D40

\footnotetext{
${ }^{1}$ Corresponding author. E-mail: jean-christophe.poggiale @ univmed.fr
} 


\section{Introduction}

It is well known that spatial structure can exhibit significant effects on spatially distributed populations or communities dynamics. These effects are various and some methods for integrating explicit spatial processes in population dynamics models have been extensively discussed (see [21], [24],[6] for instance). Spatial structures can result from biotic interactions themselves ([27], [26]) but also from environmental constraints ([25]), which shall be the case in our paper. The effects of dispersion on the stability of equilibrium has been discussed in many papers (see [4] and references therein for a detailed discussion). Roughly, effects of different factors, like dispersion rates intensity, on populations or communities dynamics have been analyzed from the individuals behavioral level ([4], [16]) or the population level ([31], [28]) to the metapopulation level ([36]), the community level ([10], [12], [22], [7], [30], [20], [25], [26]) and the ecosystem level ([23]). In most of the previous works at the population or community levels, authors are interested in the role of spatial structure on the dynamics stability. However, some works deal with other concepts like permanence ([8]) or persistence ([32], [1]). Nowadays, it is admitted that spatial heterogeneity induces a complexity in the analysis of population or community dynamics, from the observation as well as from the theoretical point of view ([34]). Some further investigations are needed to improve our knowledge on the effect of spatial structure on models analysis. In previous works ([29], [30]), we suggested a method based on time scales separation in order to simplify patchilly distributed models to analyze the effects of spatial heterogeneity on population dynamics. In our work, spatial heterogeneity means that some parameters (growth rates, predation rates, etc.) can differ from one patch to another, for some reasons. In [35] for instance, the authors analyzed the effects of spatial heterogeneity on the stability of a predator-prey system in a patchy environment where a patch was a refuge for the prey. This refuge affects the global predation rate which modifies the stability of the predator-prey dynamics. This paper illustrates namely that even in rich environments, the destabilization can be avoided and the mechanisms for this solution of the "Enrichment paradox" suggested in this article refer to the reduction of interactions intensity between prey and predator via the refuge. In [29], we studied a similar system with a predator-prey in a patchy environment and a refuge for the prey. From the ecological point of view, our results were roughly similar to those obtained in [35] but we shown how our method can be useful for providing analytical results at the global level, even in a particular case where the time scale separation application is more subtle. In [26], the authors show that an auto-organized spatial structure (spatiotemporal chaos) in a homogeneous environment can also prevent extinction of populations and resolve the paradox of enrichment.

In the present paper, we still analyze a predator-prey model in a patchy environment. We consider a vertically structured water column in which light penetration is limited. Even if nutrients do not concentrate in the immediate vicinity of the surface, it roughly results that only phytoplankton cells located high enough in the water column are able to grow thanks to the light while cells in the bottom can not (or only poorly) photosynthesize. This spatial structure of light availability induces spatial heterogeneity in phytoplankton cells population growth. Hydrodynamism in mixed layer permits to the phytoplankton cells to disperse in the water column and to take benefit from light at some times. Zooplankton also disperses in the water column and grazes on phytoplankton. 
We study how the light-induced spatial heterogeneity affects the predator-prey interactions and the resulting dynamics. We show that even in oligotrophic ecosystems, oscillations may result from the spatial heterogeneity. In [18] and [19], the authors show that coupled oscillations of daphnia and algae are common in the field. Nevertheless, the amplitudes of the oscillations are rather small and the destabilization induced by nutrient increase does not occur. However, in [11], the authors present a real phytoplankton-zooplankton system in a chemostat, which exhibits the Hopf bifurcation induced by enrichment. Thus the effect of enrichment on stability is really unclear and relations between laboratory and fields results need to be analyzed in the framework of a theory which can explain their paradoxal results. As it is already explained in [35], spatial heterogeneity probably plays a role in the fields and its effects can be complex: for instance, if results from [35] can lead to the conclusion that some aspects of heterogeneity are stabilizing, we show that other aspects are destabilizing.

Indeed, in the present paper, we explain how the destabilization can occur even for low local carrying capacities, that is in environments which are poor in nutrient, if heterogeneity is high enough. We first explain how the above mentioned conditions may lead to an effective carrying capacity of the water column larger that it should be. As a consequence, the zooplankton - phytoplancton community may exhibit oscillations even in rather poor nutrient environments. We then analyze the mechanisms under which spatial heterogeneity can lead to this result.

In the next section, we present the phytoplankton - zooplankton model. We provide a mathematical analysis in some range of the parameter values, namely when dispersion rates are very low or very high. In the former case, the analysis is based on the idea that a low intensity of dispersion rates leads to a quasi separation of patches which are studied separately. The latter case need singular perturbation techniques which are briefly recalled. In the fourth section, we complete the analytical results by some numerical studies which permit to improve our understanding of the role of spatial heterogeneity. The different factors like enrichment, heterogeneity and dispersion rates intensity are used as bifurcation parameters in order to compare homogeneous and heterogeneous situations. A conclusion ends the paper.

\section{The phytoplankton - zooplankton model}

\subsection{System description and general assumptions}

We consider a phytoplankton (prey) - zooplankton (predator) system in a two patches environment. In the water column, the vertical distribution of the phytoplankton species resources is not homogeneous. For instance, the light availability exponentially decreases with depth and the nutrients distribution depends on the hydrodynamics conditions. Roughly, the water column hydrodynamics can range from a rather well mixed to a stratified state. The former situation occurs for instance under windy conditions at the sea surface and leads to a quasi-homogeneous distribution of nutrients. The latter situation results in a heterogeneous distribution of nutrients, which are less concentrated at the surface and present a sharp concentration increase at the thermocline depth [17]. The heterogeneous spatial distributions of both light and nutrients result in a spatial 
variability in phytoplankton growth rate. The growth rate optimum depends on particular distributions of resources and there is no general consensus in the literature to situate it with precision, but, roughly, it is either very close to the surface (well mixed column) or anyway no deeper than the thermocline (stratified column). In our paper, we vertically split the water column in two patches and we assume that the limit is set under the growth rate optimum deepness, so that the growth rate in patch 1 (upper layer) is larger than the growth rate in patch 2 (deeper layer).

We then also assume that the water layer represented by patch 1 is less thick than water layer represented by patch 2 . As an example, patch 1 can represent a layer with a depth comprised between 0 and $30-40 \mathrm{~m}$ while patch 2 corresponds to a layer from $30-40 \mathrm{~m}$ to $120 \mathrm{~m}$. As a consequence, dispersion rates from patch 1 to path 2 are higher than dispersion rates from patch 2 to patch 1 . Indeed, at any time, among individuals of the thin patch 1, a high proportion are bounded close to the interface with patch 2 and are therefore susceptible to cross it downwards, whereas the proportion of individuals of patch 2 close to the interface is rather small compared to all the individuals lying in the remaining great volume of patch 2 . We insist that the hypothesis applies on the per capita dispersion rates and not on the fluxes of individuals at the interface on which we do not make any a priori assumption.

We finally make the following general assumptions for the model: on each patch, the phytoplankton population growth is governed by a logistic equation and the consumption rate by zooplankton is represented by a Holling type II functional response.

\subsection{Model description}

We consider the following model:

$$
\begin{aligned}
\frac{d x_{1}}{d \tau} & =d_{2}^{x} x_{2}-d_{1}^{x} x_{1}+\varepsilon\left(r_{1} x_{1}\left(1-\frac{x_{1}}{K_{1}}\right)-\frac{a x_{1}}{b+x_{1}} y_{1}\right) \\
\frac{d x_{2}}{d \tau} & =d_{1}^{x} x_{1}-d_{2}^{x} x_{2}+\varepsilon\left(r_{2} x_{2}\left(1-\frac{x_{2}}{K_{2}}\right)-\frac{a x_{2}}{b+x_{2}} y_{2}\right) \\
\frac{d y_{1}}{d \tau} & =d_{2}^{y} y_{2}-d_{1}^{y} y_{1}+\varepsilon\left(e \frac{a x_{1}}{b+x_{1}}-m\right) y_{1} \\
\frac{d y_{2}}{d \tau} & =d_{1}^{y} y_{1}-d_{2}^{y} y_{2}+\varepsilon\left(e \frac{a x_{2}}{b+x_{2}}-m\right) y_{2},
\end{aligned}
$$

where $x_{i}$ and $y_{i}$ are respectively the phytoplankton and zooplankton abundances on patch $i . r_{i}$ is the phytoplankton intrinsic growth rate on patch $i, K_{i}$ is the carrying capacity on patch $i, a$ is the per capita maximal ingestion rate of phytoplankton, $b$ is the half-saturation constant, that is the abundance of phytoplankton for which the ingestion rate is half the maximal ingestion rate, $e$ is the conversion efficiency and $m$ is the zooplankton natural death rate. The parameter $d_{i}^{x}$ (resp. $\left.d_{i}^{y}\right)$, where $i=1,2$, is the dispersion rate from patch $i$ to the other patch for the phytoplankton (resp. zooplankton) population. Finally, $\varepsilon$ is a dimensionless parameter which permits to account for different time scales. When $\varepsilon$ is small ( $\varepsilon \simeq 0$ ), the dispersion between patches is much faster than 
the demographic processes (growth, predation and death). According to the assumptions listed in the previous sub-section, we have : $d_{1}^{x}>d_{2}^{x}, d_{1}^{y}>d_{2}^{y}$ and $r_{1}>r_{2}$.

\section{Mathematical analysis of the model}

\subsection{Low dispersion rates}

If we assume that $\varepsilon$ is very large, the system (2.1) is a small $\frac{1}{\varepsilon}$-perturbation of the system:

$$
\begin{aligned}
\frac{d x_{1}}{d \theta} & =r_{1} x_{1}\left(1-\frac{x_{1}}{K_{1}}\right)-\frac{a x_{1}}{b+x_{1}} y_{1} \\
\frac{d x_{2}}{d \theta} & =r_{2} x_{2}\left(1-\frac{x_{2}}{K_{2}}\right)-\frac{a x_{2}}{b+x_{2}} y_{2} \\
\frac{d y_{1}}{d \theta} & =\left(e \frac{a x_{1}}{b+x_{1}}-m\right) y_{1} \\
\frac{d y_{2}}{d \theta} & =\left(e \frac{a x_{2}}{b+x_{2}}-m\right) y_{2}
\end{aligned}
$$

where $\theta=\varepsilon \tau$. This four-dimensional system is actually composed of two separated two-dimensional sub-systems, one on patch 1 and the other on patch 2 ; both of them are Rozensweig McArthur models. They are structurally stable, thus the $1 / \varepsilon$-perturbation does not affect the dynamics from the qualitative point of view. On each patch, there are three potential equilibria: $(0,0),\left(K_{i}, 0\right)$ and $\left(x_{e, i}, y_{e, i}\right)$, where $i=1$ or 2 . The equilibrium $\left(x_{e, i}, y_{e, i}\right)$ is positive only if $e a>m$ and $K_{i}>x_{e, i}$. More precisely, we have $x_{e, i}=\frac{m b}{e a-m}$ and $y_{e, i}=\frac{r}{a}\left(1-\frac{x_{e, i}}{K_{i}}\right)\left(b+x_{e, i}\right)$. The equilibrium $(0,0)$ is a saddle point for each sub-system. If $K_{i}<x_{e, i}$ on patch $i$ then the equilibrium $\left(K_{i}, 0\right)$ is globally asymptotically stable in the sense that all initial conditions with positive coordinates reach this equilibrium. If $K_{i}$ crosses the value $x_{e, i}$ then a transcritical bifurcation occurs ; when $K_{i}>x_{e, i}$, the equilibrium $\left(K_{i}, 0\right)$ is a saddle point. The equilibrium $\left(x_{e, i}, y_{e, i}\right)$ is globally asymptotically stable, in the sense given above, if $K_{i}<b+2 x_{e, i}$. Let $K_{i, c}=b+2 x_{e, i}$ : this value is a Hopf bifurcation value of the local carrying capacity parameter. If $K_{i}>K_{i, c}$ then the positive equilibrium $\left(x_{e, i}, y_{e, i}\right)$ is unstable and the trajectories initiated in the positive quadrant reach a limit cycle. In the present manuscript, we will now assume that on each patch $x_{e, i}<K_{i}$ which means that, separately, both sub-systems have a positive equilibrium which is globally asymptotically stable if $K_{i}<K_{i, c}$. It follows that when the dispersion is very low and if $K_{i}<K_{i, c}$, the system (2.1) exhibits a globally asymptotic stable equilibrium (details of this analysis can be found in [5] for instance); this result is illustrated on figure (1). 

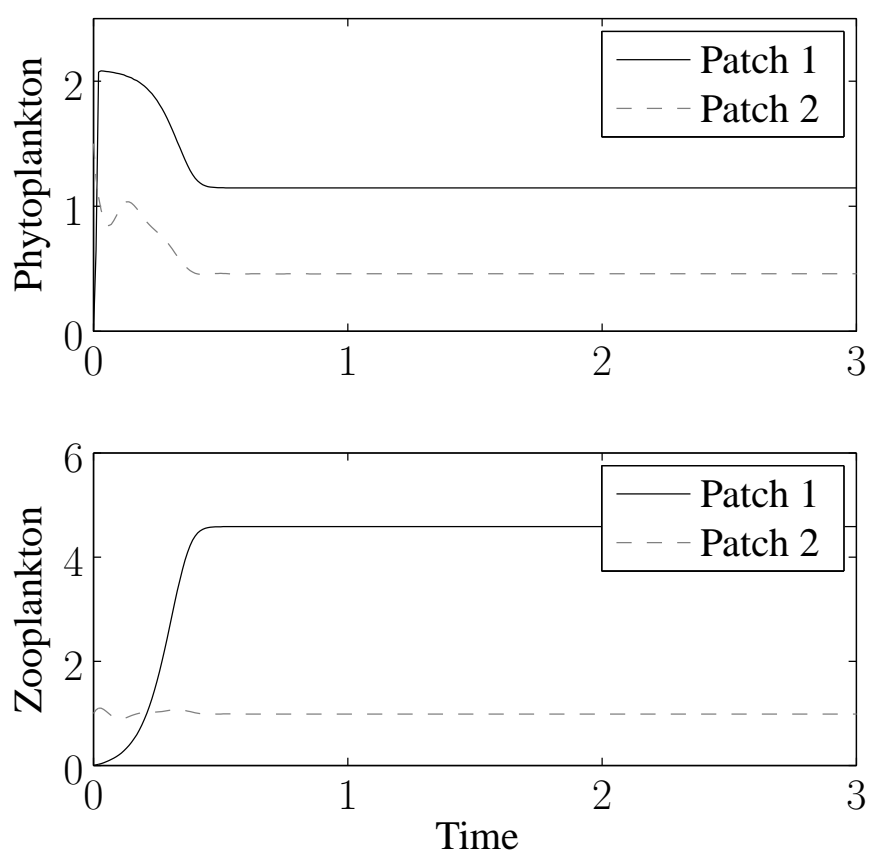

Figure 1: This figure illustrates the dynamics of the phytoplankton and of the zooplankton on each patch with model (2.1), for low dispersion rates. On each graphics, there are two curves. The black lines exhibit the patch 1 dynamics, the dashed grey ones show the patch 2 dynamics. The parameters values are the following: $r_{1}=6$; $r_{2}=1 ; K_{1}=2.1 ; K_{2}=2.1 ; a=1.2 ; b=0.9 ; d_{1}^{x}=4 ; d_{2}^{x}=1 ; d_{1}^{y}=4 ; d_{2}^{y}=1 ; e=0.8 ; m=0.5 ;$ $\varepsilon=100$.

\subsection{High dispersion rates : Aggregation of variables on the water column}

If we assume that $\varepsilon$ is small, we can use the singular perturbation theory in order to aggregate the phytoplankton population and the zooplankton population on the whole water column. In other words, let us consider $x=x_{1}+x_{2}$ the total phytoplankton population abundance and $y=y_{1}+y_{2}$, the total zooplankton population abundance, then we can write a two-dimensional model governing these global variables (see [2] for a detailed explanation and examples of the method). In order to get this model, we first consider the non pertubated system by letting $\varepsilon=0$ in system (2.1):

$$
\begin{aligned}
& \frac{d x_{1}}{d \tau}=d_{2}^{x} x-\left(d_{1}^{x}+d_{2}^{x}\right) x_{1} \\
& \frac{d y_{1}}{d \tau}=d_{2}^{y} y-\left(d_{1}^{y}+d_{2}^{y}\right) y_{1} \\
& \frac{d x}{d \tau}=0 \\
& \frac{d y}{d \tau}=0 .
\end{aligned}
$$

According to this system, the global variables $x$ and $y$ are constant and the vector $\left(x_{1}, y_{1}, x, y\right)$ 
tends to $\left(u_{1} x, v_{1} y, x, y\right)$, where $u_{1}$ and $v_{1}$ are the proportion located in patch 1 of the preys and predators in the whole column, at equilibrium. After a short transient time, the fast dispersion leads to a constant frequency of prey and predator populations on patch 1 . The frequencies are:

$$
\begin{aligned}
& u_{1}=\frac{d_{2}^{x}}{d_{1}^{x}+d_{2}^{x}} ; \quad u_{2}=1-u_{1} \\
& v_{1}=\frac{d_{2}^{y}}{d_{1}^{y}+d_{2}^{y}} ; \quad v_{2}=1-v_{1}
\end{aligned}
$$

We can reduce the previous four-dimensional system (2.1) to the following two-dimensional system:

$$
\begin{aligned}
& \frac{d x}{d t}=r x\left(1-\frac{x}{K}\right)-a\left(\frac{u_{1} v_{1}}{b+u_{1} x}+\frac{u_{2} v_{2}}{b+u_{2} x}\right) x y \\
& \frac{d y}{d t}=\left(e a\left(\frac{u_{1} v_{1}}{b+u_{1} x}+\frac{u_{2} v_{2}}{b+u_{2} x}\right) x-m\right) y,
\end{aligned}
$$

where $t=\varepsilon \tau, r=r_{1} u_{1}+r_{2} u_{2}$ and $K=\frac{r K_{1} K_{2}}{r_{1} u_{1}^{2} K_{2}+r_{2} u_{2}^{2} K_{1}}$. The functional response at the global level is thus:

$$
G(x)=g(x) x
$$

where

$$
g(x)=a\left(\frac{u_{1} v_{1}}{b+u_{1} x}+\frac{u_{2} v_{2}}{b+u_{2} x}\right) .
$$

The equilibria of this system are: $E_{1}=(0,0), E_{2}=(K, 0)$ and, when $m<e a, E_{3}=\left(x_{e}, y_{e}\right)$, where $x_{e}$ is the unique solution of the equation:

$$
G(x)=\frac{m}{e} .
$$

If $m>e a$, this equation has no solution. A straightforward algebraic manipulations leads to:

$$
\begin{aligned}
x_{e} & =\frac{-b\left(u_{1} v_{1}+u_{2} v_{2}-\frac{m}{e a}\right)+\sqrt{\Delta}}{2\left(1-\frac{m}{e a}\right) u_{1} u_{2}} \\
\text { where } \Delta & =b^{2}\left(\left(u_{1} v_{1}+u_{2} v_{2}-\frac{m}{e a}\right)^{2}+4 \frac{m}{e a}\left(1-\frac{m}{e a}\right) u_{1} u_{2}\right) .
\end{aligned}
$$

Moreover, $y_{e}$ is defined by:

$$
y_{e}=r \frac{\left(1-\frac{x_{e}}{K}\right)}{g\left(x_{e}\right)}
$$

thus $y_{e}$ is positive only if $K>x_{e}$. The functional response $G=x . g$ satisfies the following properties, for all $x \geq 0$ :

$$
\begin{aligned}
G(x) & \geq 0 \\
G^{\prime}(x) & >0 \\
g^{\prime}(x) & <0 .
\end{aligned}
$$


Then the stability of $E_{3}$ is obtained if the isocline $y=r \frac{\left(1-\frac{x}{K}\right)}{g(x)}$ is decreasing at the equilibrium. In other words, $E_{3}$ is stable if the derivative of the function:

$$
x \longmapsto r \frac{\left(1-\frac{x}{K}\right)}{g(x)}
$$

is negative at $x=x_{e}$. This leads to the following conditions:

$$
0<-\left(1-\frac{x_{e}}{K}\right) g^{\prime}\left(x_{e}\right)<\frac{m}{e K x_{e}}
$$

It is easy to write the previous condition with respect to the carrying capacity $K$ :

$$
K<x_{e}-\frac{m}{e x_{e} g^{\prime}\left(x_{e}\right)}
$$

Let $K_{c}=x_{e}-\frac{m}{e x_{e} g^{\prime}\left(x_{e}\right)}$. If $K<K_{c}$, then the positive equilibrium is locally stable, while if $K>K_{c}$, then the positive equilibrium is locally unstable and a stable limit cycle appears. $K_{c}$ is a Hopf bifurcation value of the carrying capacity parameter. Figure (2) illustrates the similarity between the complete model (2.1) and the reduced one (3.5).

\subsection{High dispersion rates : Comparison between homogeneous and hetero- geneous situations}

We show in this subsection that the spatial heterogeneity induced by light and described by differences in the $r$ and $K$ local values, can lead to oscillations which would not occur in the homogeneous case. More precisely, we shall compare the situation where $r_{1} \neq r_{2}$ (heterogeneous case) to the situation where $r_{1}=r_{2}$ (homogeneous case) and we show that, with the above listed assumptions and for a given set of parameters, the homogeneous case can lead to a globally asymptotically stable equilibrium while the heterogeneous case can exhibit oscillations.

In [28], we considered a system of one population living on two patches with high dispersion rate and for which the growth on each patch was governed by logistic equations. In that paper, the local carrying capacities $K_{1}$ and $K_{2}$ were assumed to be the same: $K_{1}=K_{2}$. We shown that the total population was following a logistic growth too. Moreover, in the heterogeneous situation, which was represented by $r_{1} \neq r_{2}$, we shown that the global carrying capacity could be larger than $K_{1}+K_{2}=2 K_{1}$, in consequence of the nonlinearities of growth in a variable environment. Suppose $r_{1}>r_{2}$ (in the opposite case, the inequalities must be changed by permuting the indexes), the established condition to get the result was:

$$
\frac{r_{1}}{r_{2}}>\frac{d_{1}^{x}}{d_{2}^{x}}>1
$$

The result can be extended to the situation where $K_{1} \neq K_{2}$. The condition becomes:

$$
\frac{K_{2} r_{1}}{K_{1} r_{2}}>\frac{d_{1}^{x}}{d_{2}^{x}}>\frac{K_{2}}{K_{1}}
$$



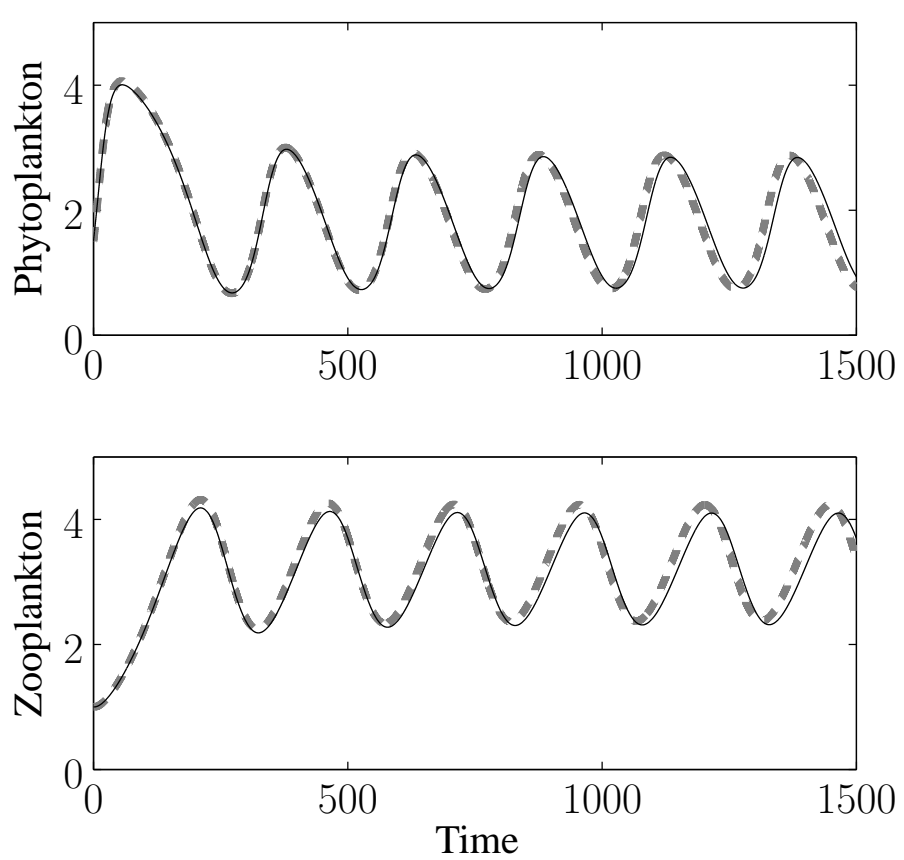

Figure 2: This figure illustrates the dynamics of the phytoplankton and of the zooplankton in the whole system, in heterogeneous conditions. On each graphics, there are two curves. The black lines are obtained with the complete model (2.1) while the dashed grey ones result from the aggregated model (3.5). The parameters values are the following: $r_{1}=6 ; r_{2}=1 ; K_{1}=2.1 ; K_{2}=2.1 ; a=1.2 ; b=0.9 ; d_{1}^{x}=4 ; d_{2}^{x}=1 ; d_{1}^{y}=4 ; d_{2}^{y}=1$; $e=0.8 ; m=0.5 ; \varepsilon=0.05$.

and the proof is the same as that in [28]. Let us assume that these inequalities are satisfied. We thus know that the total carrying capacity $K$ of the phytoplankton population can be enlarged by the spatial heterogeneity induced by the differences between the growth rates on both patches. However, the bifurcation value $K_{c}$ is the same in the homogeneous and in the heterogeneous case since it does not depend on $r_{i}$ and $K_{i}$. It follows that in the heterogeneous situation, the global carrying capacity can be larger than $K_{c}$ while it is not the case in the homogeneous situation with the previous assumptions. As a consequence, spatial heterogeneity can lead to a destablization of the positive equilibrium and may result in the occurrence of populations fluctuations. This result can be illustrated by comparing figures (2) and (3). Indeed, these figures are obtained with the same parameter values excepted for the parameter $r_{1}$. On figure (2), parameter $r_{1}$ is 6 (that is $r_{1}>r_{2}$ ) what corresponds to a heterogeneous environment while on figure (3), $r_{1}=r_{2}$, what corresponds to a homogeneous environment. 

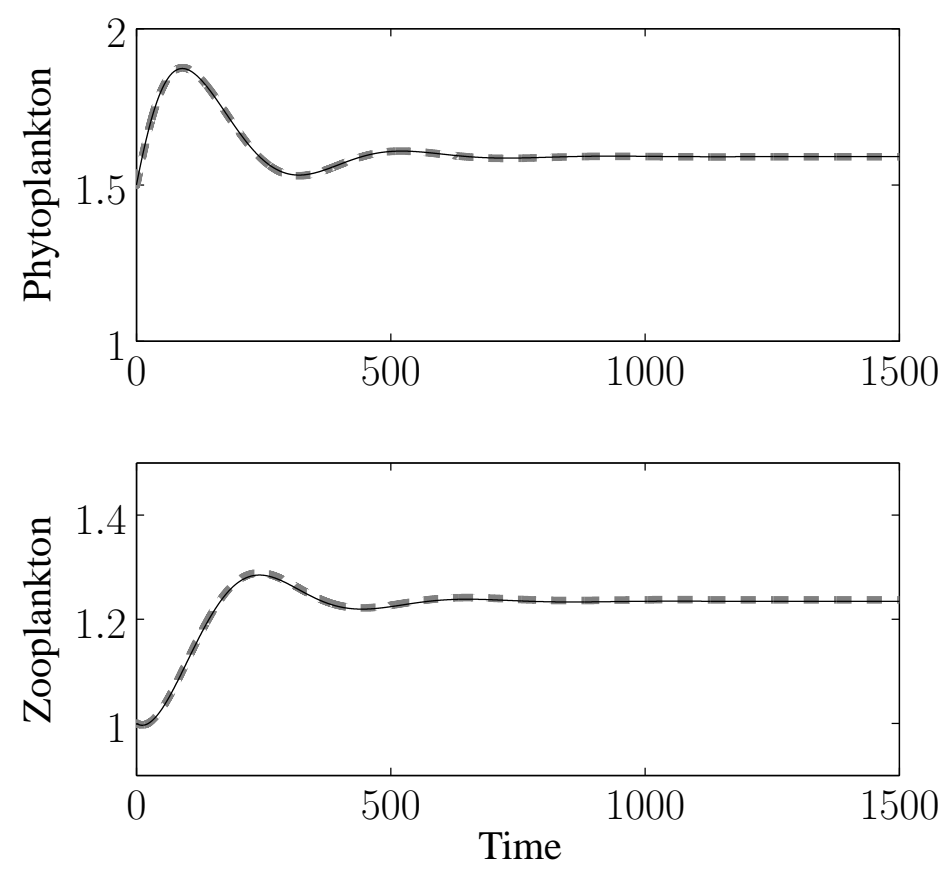

Figure 3: This figure illustrates the dynamics of the phytoplankton and of the zooplankton in the whole system, in homogeneous conditions. On each graphics, there are two curves. The black lines are obtained with the complete model (2.1) while the dashed grey ones result from the aggregated model (3.5). The parameters values are the following: $r_{1}=1 ; r_{2}=1 ; K_{1}=2.1 ; K_{2}=2.1 ; a=1.2 ; b=0.9 ; d_{1}^{x}=4 ; d_{2}^{x}=1 ; d_{1}^{y}=4 ; d_{2}^{y}=1$; $e=0.8 ; m=0.5 ; \varepsilon=0.05$.

\section{Intermediate dispersion rates and general numerical results}

In this section, we extend the previous results to intermediate dispersion rates. As we shown in the previous section, we could deal with different $K_{i}$ on both patches, which would be more realistic. However, for the sake of simplicity, we decided to set $K_{1}=K_{2}:=K$, what still let us explore the effects of spatial heterogeneity of local resources richness but through one single parameter $\left(r_{i}\right)$. We investigate the role of spatial heterogeneity, dispersion rates intensity and resources richness on the stability of the system, which is here represented by the local stability of the positive equilibrium, with model (2.1). In order to perform this analysis, we proceed in three steps. In the first step, we analyze the stability of the predator-prey model by means of a bifurcation diagram in the richness - dispersal plane for two extreme environment conditions: homogeneous and heterogeneous. The second step deals with the analysis of the effect of dispersion rates on the structure of the bifurcation diagram in the heterogeneity index - local carrying capacity plane. Finally, we study the effect of the local carrying capacity on the structure of the bifurcation diagram in the dispersal - heterogeneity index plane. The bifurcation analyses were performed using continuation technics by means of the software (matlab toolbox) Matcont [9]. 


\subsection{Effect of spatial heterogeneity}

We first compare the effect of enrichment and dispersion rates intensity in two types of environments ; one is homogeneous while the second is heterogeneous. The homogeneous situation is obtained by setting $r_{1}=r_{2}$, the heterogeneous case satisfies condition (3.6). In both cases, we determined the bifurcation diagram in the $(K, \varepsilon)$-parameter space. The difference between the homogeneous case and the heterogeneous case, illustrated on figure (4) is important. In the former case, the system has a stable positive equilibrium for low carrying capacity, whatever $\varepsilon$ value is, that is whatever the intensity of dispersion is, in the range we considered. In the latter case, the system has a stable positive equilibrium for lower dispersion rates, whatever the carrying capacity is in the range we considered. From the ecological point of view, it means that a Hopf bifurcation occurs in the homogeneous case when the carrying capacity increases, that is by enrichment. However, in the heterogeneous case, the Hopf bifurcation needs less enrichment to occur when the dispersion rates increase ( $\operatorname{small} \varepsilon$ ), that is when the mixing induced by hydrodynamics processes is intensified.
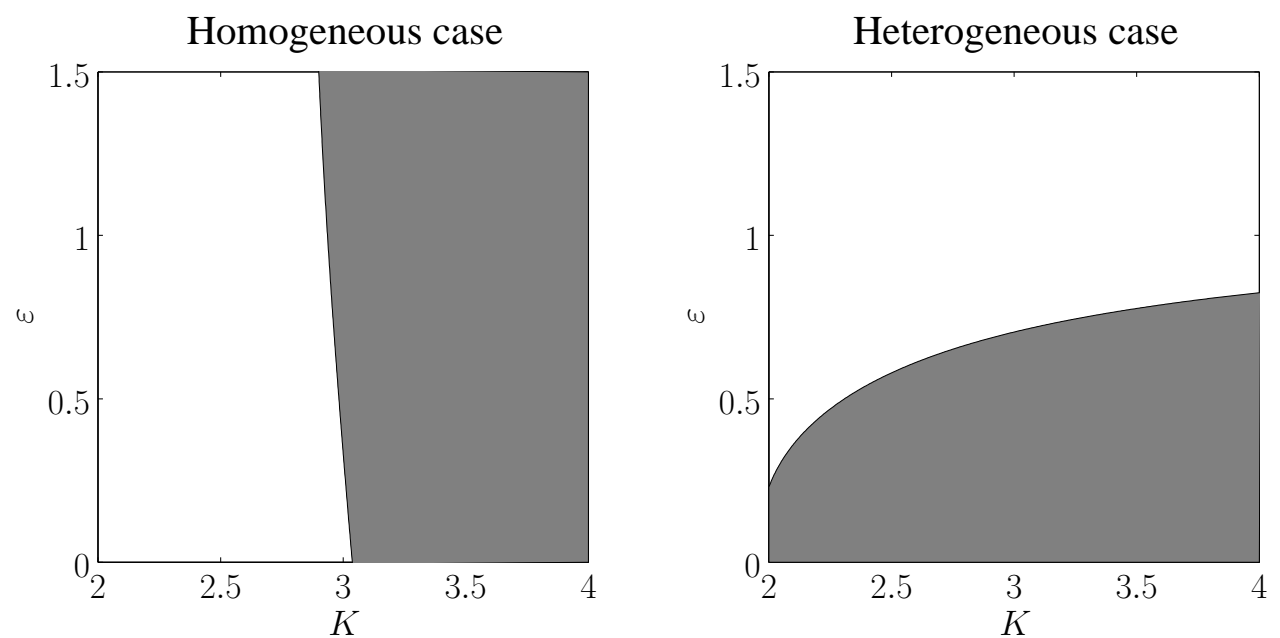

Figure 4: On this figure, an homogeneous case (left) and an heterogeneous case (right) are compared. They are simulated with model (2.1). The homogeneous case corresponds to identical prey growth rates $r_{1}=r_{2}$ (and we set these parameters to 1 ) and the heterogeneous case corresponds to $r_{1} \neq r_{2}$. In this case, we set $r_{1}=6$ and $r_{2}=1$. We compare these situations for various local carrying capacities and various dispersion rates intensities. The white regions, the "stability regions", indicate the existence of a stable equilibrium ; gray regions correspond to "unstability regions" where no stable equilibrium exists (the same color code will be kept in the figures that follow). The figure illustrates that the main bifurcation parameter involved in the destabilization (Hopf bifurcation) is the carrying capacity for the homogeneous case whereas it is the dispersion intensity rates for heterogeneous case. Note the persistence of the unstability region for low values of $K$ in the heterogeneous case. The parameters values are the following: $K_{1}=2.1 ; K_{2}=2.1 ; a=1.2 ; b=0.9 ; d_{1}^{x}=4 ; d_{2}^{x}=1$; $d_{1}^{y}=4 ; d_{2}^{y}=1 ; e=0.8 ; m=0.5$. $\varepsilon$ varies from 0.01 to 1 . 


\subsection{Effect of dispersion rates intensity}

In order to understand how the spatial heterogeneity affects the organization of these bifurcation diagrams, we define an heterogeneity index by the ratio of the growth rates: $\alpha=\frac{r_{1}}{r_{2}}$. The homogeneous case corresponds to $\alpha=1$ and spatial heterogeneity increases with $\alpha$. Even if spatial heterogeneity increases when $\alpha$ decreases from 1 to 0 , we do not consider this situation which does not lead to the condition (3.6). Figure (5) illustrates the result by means of bifurcation diagrams in the parameter space $(K, \alpha)$, for different dispersion rates intensities measured by $\varepsilon$. We can see on the left panel of figure (5), obtained for high dispersion rates $(\varepsilon=0.05)$, that when the heterogeneity index $\alpha$ increases, the region of stability decreases, enhancing the result that, for a given resources richness, heterogeneity can have a destabilizing effect and can generate fluctuations in populations densities. Figure (5) shows how this destabilization is organized when spatial heterogeneity increases, for various dispersion intensities. We can see that the effect of spatial heterogeneity is more important for high dispersion rates since when these rates decrease ( $\varepsilon$ increases), the dependence of the bifurcation diagram on the heterogeneity index tends to disappear and the region of stability of the positive equilibrium spreads throughout the bifurcation diagram. It is noticeable that for these intermediate dispersion rates, the carrying capacity needed to destabilize the system is very high, even in the homogeneous situation. The stabilizing effect of dispersal is highlighted here. Increasing $\varepsilon$ means decreasing the dispersion rates, which leads to an isolation of patches and the local parameters are such that on each isolated patch, the population densities reach equilibrium values. It follows that for very high value of $\varepsilon$, the bifurcation diagram should not depend on $\alpha$ and should exhibits a behavior, with respect to $K$, similar to what is expected on each patch when isolated. This situation is only obtained for very high values of $\varepsilon$.
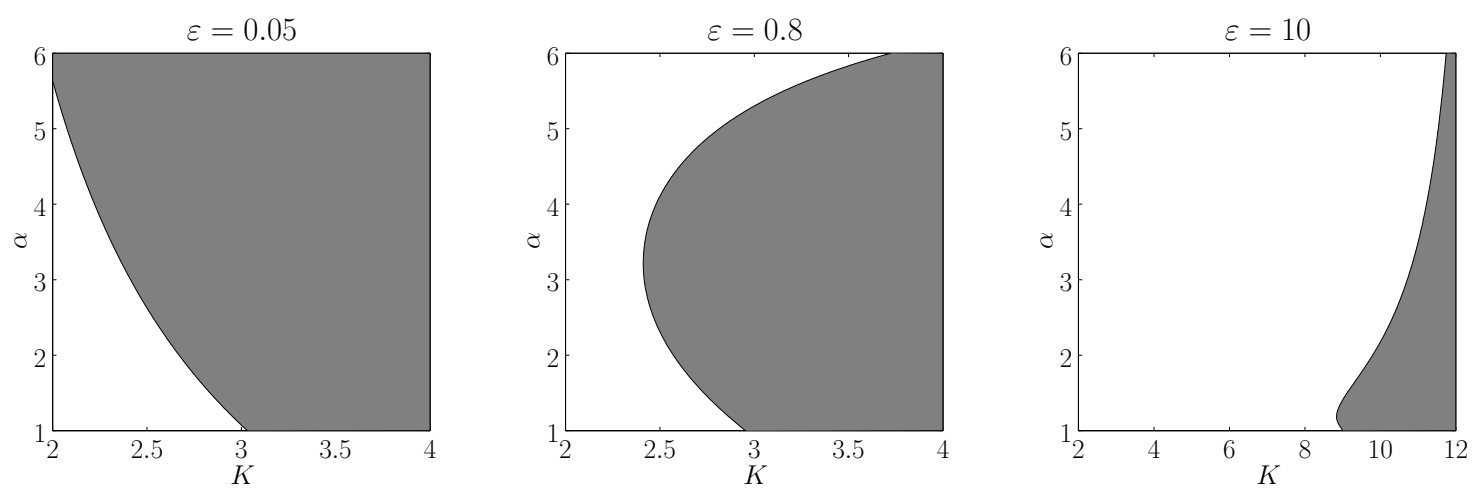

Figure 5: In this figure, we represent the destabilizing effect induced by an increased carrying capacity according to the ratio of the growth rates $\alpha=\frac{r_{1}}{r_{2}}$, which is a measure of spatial heterogeneity. The three panels correspond to different levels of dispersion rates, from very fast dispersion (left, $\varepsilon=0.05$ ), intermediate dispersion rate (middle, $\varepsilon=0.8$ ), to slow dispersion (right, $\varepsilon=10$ ). Note the enlarged scale of the $\mathrm{x}$-axis in the right panel. The left-panel figure illustrates the enlargement of the unstability region when the heterogeneity increases, for high dispersion rates. As the dispersion rate gets lower (intermediate and slow dispersion), the effect of spatial heterogeneity diminishes, while the stability region fills up the diagram (right panel). Parameters values are the following: $r_{2}=1 ; a=1.2 ; b=0.9 ; d_{1}^{x}=4 ; d_{2}^{x}=1 ; d_{1}^{y}=4 ; d_{2}^{y}=1 ; e=0.8 ; m=0.5$. 


\subsection{Effect of local carrying capacity}

In this last step, we analyze a bifurcation diagram in the $(\varepsilon, \alpha)$-parameter space, see figure (6). A small region of unstability exists for very high dispersion rates (small $\varepsilon$ ) and a large region of unstability exists for low dispersion rates. These regions are separated by a large region of stability. Let us note that the chosen local carrying capacity value is such that if the patches were isolated, the dynamics on each patch would exhibit limit cycles. It follows that for a given heterogeneity index, the system is unstable for very high dispersion rates. Intermediate dispersion rates stabilize the predator-prey system. The very low dispersion rates case looks like a system of isolated patches, with fluctuations. The width of the stability region is greater for high values of the heterogeneity index $\alpha$.

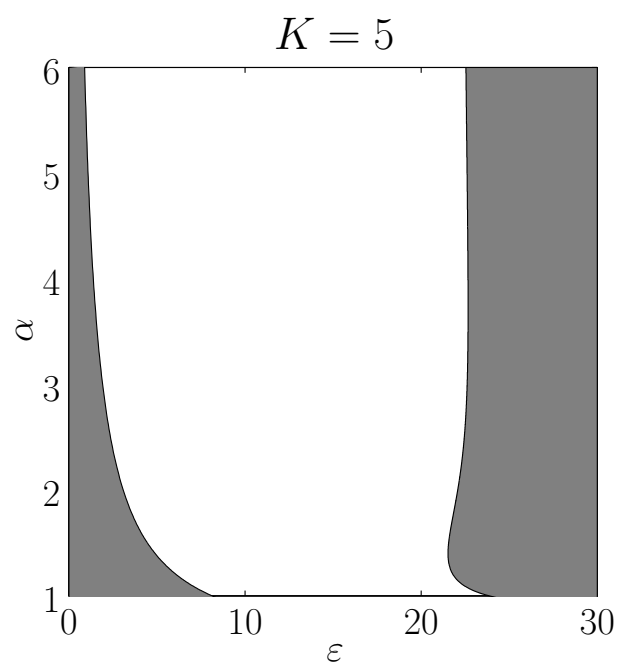

Figure 6: This figure illustrates the combined effects induced by spatial heterogeneity, measured with $\alpha=\frac{r_{1}}{r_{2}}$ and dispersion rates intensity, given by $\varepsilon$. The other parameters values are the following: $r_{2}=1 ; a=1.2$; $b=0.9 ; d_{1}^{x}=4 ; d_{2}^{x}=1 ; d_{1}^{y}=4 ; d_{2}^{y}=1 ; e=0.8 ; m=0.5 ; K=5$. For greater values of $K$, the stability region would enlarge, while the right hand unstability region would disappear if $K$ gets smaller.

A systematic study with regards to variations of $K$ let us precise the behavior of the system (unshown result): when the local carrying capacity $K$ increases, the stability region narrows down and only a small window of dispersion rates values can stabilize the system; at the opposite, when $K$ gets too small, the high- $\varepsilon$ unstability region disappears, what corresponds to the expected regime of equivalent isolated patches.

\section{Conclusion}

According to previous works like [35] or [29], for instance, spatial heterogeneity affects the stability of predator-prey systems. However, we illustrated how this factor acts in a complex way and 
leads to apparent contradictory effects. We associated it with two other factors, namely the dispersion rates intensity and the resources richness, in order to see how they all interact and to dispell this contradiction in enlightening its origin. These two other factors have been chosen because the literature also largely associates them with stability issues in predator-prey systems. For instance, in chemostat, enrichment can lead to destabilization, as authors shown experimentally in [11].

Moreover, as we mentioned in the introduction, some works like [13] exhibited the stabilizing effect of dispersal. However, in this work, even if there is a heterogeneous distribution of populations induced by predator-prey interactions, the environment is homogeneous. We shown here that spatial heterogeneity can reduce or enhance this stabilizing strength of dispersion. In a real marine system, dispersion rates can be very low as well as very high according to hydrodynamics constraints. We therefore analyzed the extreme situations analytically by means of aggregation techniques for instance and we extended the results to intermediate situations by using numerical bifurcation analysis. These approaches were already combined in previous works ([3] for instance) and this combination reveals itself to be efficient for understanding the dynamics of rather high dimension nonlinear systems.

\section{Acknowledgements}

The authors are grateful to the two anonymous referees for their remarks which helped to improve a first version of this paper.

\section{References}

[1] F.R Adler, Migration alone can produce persistence of host-parasitoid models, Am. Nat., 141 (1993), 642-650

[2] P. Auger, R. Bravo de la Parra, J.-C. Poggiale, E. Sanchez, and T. Nguyen-Huu, Aggregation of Variables and Applications to Population Dynamics in Structured Population Models in Biology and Epidemiology Lect. Notes in Math., Mathematical Biosciences Subseries , (2008), Vol. 1936 P. Magal, S. Ruan, (Eds.), 345 p.

[3] P. Auger, B.W. Kooi, R. Bravo de la Parra and J.-C. Poggiale, Bifurcation analysis of a predator-prey model with predators using hawk and dove tactics J. Theor. Biol., 238, (2006), 597-607

[4] C. Bernstein, P. Auger and J.-C. Poggiale, Predator Migration decisions, the Ideal Free Distribution and predator-prey dynamics, Am. Nat., 153, (1999), Vol. 3, 267-281

[5] F. Brauer, C. Castillo-Chavez, Mathematical models in Population Biology and Epidemiology, Texts in Applied Matheamtics 40, Spinger - Verlag, (2000)

[6] R.S. Cantrell, and C. Cosner, Spatial Ecology via Reaction - Diffusion Equations, Wiley Series in Mathematical and Computational Biology, Wiley, (2003) 
[7] P.H. Crowley, Dispersal and the stability of predator-prey interactions, Am. Nat., 118, (1981), 673-701

[8] J. Cui, The Effect of Dispersal on Permanence in a Predator-Prey Population Growth Model, Comp. Math. Appl., 44, (2002), 1085-1097

[9] A. Dhooge, W. Govaerts and Y.A. Kuznetsov, MATCONT : a Matlab package for numerical bifurcation analysis of ODEs, ACM Transactions on Mathematical Software 29, (2003), 141164

[10] K.E. Dyson, M.T. Bulling, M. Solan, G. Hernandez-Milian, D.G. Raffaelli, P.C.L. White, D.M. Paterson, Influence of macrofaunal assemblages and environmental heterogeneity on microphytobenthic production in experimental systems, Proc. R. Soc. B, 274, (2007), 25472554

[11] G.F. Fussmann, S.P. Ellner, K.W. Shertzer, N.G. Hairston Jr, Crossing the Hopf Bifurcation in a Live Predator-Prey System, Science, 17, (2000), 1358-1360

[12] I. Hanski, Coexistence of competitors in patchy environments with and without predation, Oikos 37, (1981), 306-312

[13] M.P. Hassell, H.N. Comins and R.M. May, Species coexistence and self-organizing spatial dynamics, Nature, 370, (1994), 290-292

[14] M.P. Hassel and R.M. May, Stability in insect host - parasite models, J. An. Ecol. 42, (1973), 693-726

[15] L. Ignatiades, S. Psarra, V. Zervakis, K. Pagou, E. Souvermezoglou, G. Assimakopoulou, and O. Gotsis-Skretas, Phytoplankton size-based dynamics in the Aegean Sea (Eastern Mediterranean), J. Mar. Syst., 36, (2002), 11-28

[16] A.R. Ives, Density dependent and density independent parasitoid aggregation in model hostparasitoid systems, Am. Nat., 140, (1992), 912-937

[17] K.H. Mann and J.R.N. Lazier, Dynamics of Marine Ecosystems: Biological - Physical Interactions in the Oceans, (1996), Blackwell Science.

[18] E. McCauley, and W.W. Murdoch, Cyclic and stable populations: plankton as a paradigm, Am. Nat., 129, (1987), 98-121

[19] E.F. McCauley, and W.W. Murdoch, Predator-prey dynamics in environments rich and poor in nutrients, Nature, 343, (1990), 455-457

[20] R. Mchich, P. Auger and J.-C. Poggiale, Effect of predator density dependent dispersal of prey on stability of a predator-prey system, Math. Biosc., 206, (2007), 343-356

[21] J.D. Murray, Mathematical Biology, Biomathematics Texts 1, (1989), vol. 9, Springer-Verlag 
[22] W.W. Murdoch and A.S. Oaten, Aggregation by parasitoids and predators : effects on equilibrium and stability, Am. Nat., 134, (1989), 288-310

[23] E.J. Murphy, J.L. Watkins, P.N. Trathan, K. Reid, M.P. Meredith, S.E. Thorpe, N.M. Johnston, A. Clarke, G.A Tarling, M.A. Collins, J. Forcada, R.S. Shreeve, A. Atkinson, R. Korb, M.J. Whitehouse, P. Ward, P.G. Rodhouse, P. Enderlein, A.G. Hirst, A.R. Martin, S.L. Hill, I.J. Staniland, D.W. Pond, D.R. Briggs, N.J. Cunningham, and A.H. Fleming, Spatial and temporal operation of the Scotia Sea ecosystem: a review of large-scale links in a krill centred food web, Phil. Trans. R. Soc., B, 362, (2007), 113-148

[24] A. Okubo and S.A. Levin, Diffusion and Ecological Problems, Mathematical Biology, Interdisciplinary Applied Mathematics, 2nd Edt, (2000), Springer

[25] M. Pascual, and H. Caswell, Environmental heterogeneity and biological patterns in a chaotic predator-prey system, J. Theor. Biol., 185, (1997), 1-13

[26] S.V. Petrovskii, B.-L. Li and H. Malchow, Transition to spatiotemporal chaos can resolve the paradox of enrichment, Ecol. Compl., 1, (2004), 37-47

[27] S.V. Petrovskii, and H. Malchow, A minimal model of pattern formation in a prey-predator system, Math. Comp. Model., 29, (1999), 49-63

[28] J.-C. Poggiale, P. Auger, D. Nrini, C. Mant and F. Gilbert, Global production increased by spatial heterogeneity in a population dynamics model, Actabioth., 53, (2005), 359-370

[29] J.-C. Poggiale and P. Auger, Impact of spatial heterogeneity on a predator-prey system dynamics C. R. Biol., 327, (2004), 1058-1063

[30] J.-C. Poggiale, Predator-Prey Models in Heterogeneous Environment : Emergence of Functional Response, Math. Comp. Model., 27, (1998), Vol. 4, 63-71

[31] J.-C. Poggiale, From Behavioural to Population level : growth and competition Math. Comp. Model., 27, (1998), Vol. 4, 41-50

[32] J. Reeve, Environmental variability, migration and persistence in host-parasitoid systems Am. Nat., 118, (1988), 810-836

[33] S. Roya and J. Chattopadhyay, Towards a resolution of 'the paradox of the plankton': A brief overview of the proposed mechanisms, Ecol. Compl., 4, (2007), 26-33

[34] D.L. Strayer, H.A. Ewing, and S. Bigelow, What kind of spatial and temporal details are required in models of heterogeneous systems?, OIKOS, 102, (2003), 654-662

[35] M. Scheffer and R.J. De Boer, Implications of spatial heterogeneity for the paradox of enrichment, Ecol., 76, (1995), 7, 2270-2277

[36] B.K. Singh, J.S. Rao, R. Ramaswamy and S. Sinha, The role of heterogeneity on the spatiotemporal dynamics of host - parasite metapopulation, Ecol. Model., 180, (2004), 435-443 